\title{
Die Kraft der Kräuterhexe
}

\section{Adrian Ritter}

Freier Journalist

\begin{abstract}
Als ihre Tochter an Krebs erkrankte, erfand Regula Meier eine Kraftfigur für sie. In einem Buch beschreibt sie jetzt, wie dies ihrer Tochter und der ganzen Familie in der schwierigen Zeit geholfen hat. Ein Stein, der mir Flügel macht ist ein Plädoyer für die Kraft der Phantasie - und eine kindgerechte Erziehung.
\end{abstract}

An einem stürmischen Januartag 2012 sass Regula Meier auf dem Bett ihrer Tochter Marina im Kinderspital Zürich. Das sechsjährige Mädchen litt an einem Neuroblastom und musste die erste Chemotherapie über sich ergehen lassen. Gemeinsam sahen Mutter und Tochter vor dem Fenster den Wind an den Bäumen zerren. Den starken Bäumen konnte der Sturm nichts anhaben, aber die schwachen wurden zu Boden gedrückt. «In diesem Moment wurde mir klar: Wir müssen so stark werden wie die unbeugsamen Bäume», beschreibt Regula Meier den entscheidenden Moment in ihrem Buch Ein Stein, der mir Flügel macht. Wie meine krebskranke Tochter der Kräuterhexe begegnete.

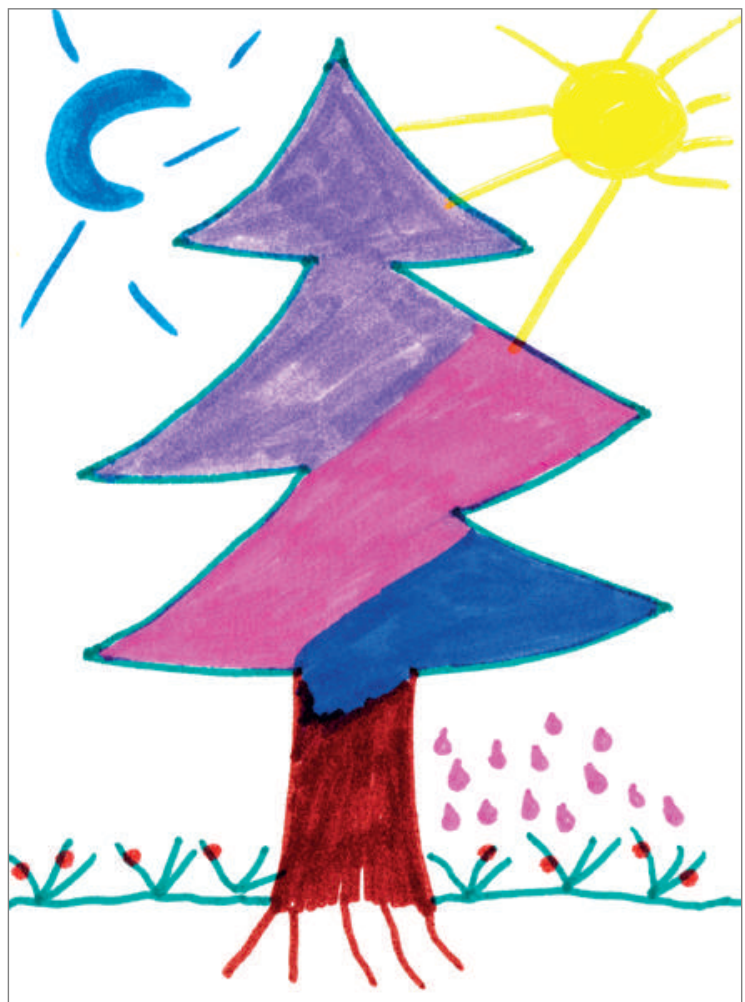

Für Regula Meier war in jenem Moment klar: «Marina brauchte einen Stern, an dem sie sich orientieren konnte, etwas, das sie motivierte. Medizinisch konnten wir unserer Tochter nicht helfen, aber wir beschlossen, dafür zu sorgen, dass es ihr psychisch besser ginge.»

Regula Meier erinnerte sich an die wilden Phantasiegeschichten ihres Vaters. Und an die Rückenoperation, wegen der sie selber mit 17 Jahren wochenlang im Bett verharren musste. Sie überstand die schwierige Zeit nicht zuletzt, indem sie sich selber Geschichten ausdachte. Deshalb erweckte sie für ihre Tochter Marina die Phantasiefigur Chrisanthemia zum Leben, die Kräu-

\section{Meine liche Mamina}

das ist mine Zaubertame. Sie stht an inem ganz speziellen Kraftort im Wald. Deine Tropten beskhen aus dem Tannensalt, spezzielten Gräsern, roken Beren Sonnerstrahlen und Mondlicht. Diese Tropken machen Dich so start wie meine Zaubertanne. Nimm jeden Tag 13 davon. Für den Appetit ist cler kraftstein. Halke inn in oler Hand und Du wirst spirren wie des Hunjer koumt. The werde jetzt immer be Dir sein. Dich spuiren und aut elich aupassen. ICh heisse Chmisanthemia und bin Deine kräkeshexe.

Die erste Botschaft der Kräuterhexe: Medizinisch helfen konnten die Eltern nicht, aber sie beschlossen, ihr krankes Kind mithilfe der Phantasie zu unterstützen. 
terhexe. Da sich Marina im Wald sehr wohl fühlte, schien ihr die Figur genau passend zu sein.

\section{Kraftsteine und Salbei-Spray}

Mit einem Brief, Krafttropfen, die stark machen, und einem Kraftstein, um den Appetit nicht zu verlieren, meldete sich Chrisanthemia kurz darauf ein erstes Mal bei Marina. Das Strahlen auf dem Gesicht der Tochter und die wiedererwachte Lebendigkeit schoben bei Regula Meier alle Zweifel beiseite, ob «dieser Schwindel überhaupt erlaubt sei».

Das im September 2015 erschienene Buch von Regula Meier beschreibt eindrücklich und bewegend, wie die Kräuterhexe half, wenn Marina fast verzweifelte und unter starken Schmerzen oder Nebenwirkungen der Therapie litt. Chrisanthemia schickte Marina Briefe und etwa einen "Mutduft» zum Einreiben oder einen Salbei-Spray gegen die Aphten im Mund während einer schweren Infektion.

Marina wiederum bastelte für die Kräuterhexe Geschenke und formulierte ihre Ängste in ihren Briefen, die sie der Mutter diktierte. «Die Kräuterhexe machte Marina stark. Sie entwickelte Kraft und Durchhaltewillen, um bei der Therapie mitzumachen. Und durch die Kräuterhexe haben Marina und wir die Angst vor dem Tod verloren", beschreibt es Regula Meier.

All dies war leider nötig, denn nach Zeiten der Stabilisierung, in denen Marina sogar den Kindergarten besuchen konnte, kehrte der Tumor mit aller Wucht zurück. Im Februar 2013 war klar: Marina würde sterben. Eine weitere Hochdosis-Chemo hätte nur geringe Chancen auf Heilung, aber enorme Nebenwirkungen. Im Gespräch mit der Palliativärztin des Kinderspitals Zürich entschieden sich die Eltern für den Weg der palliativen Behandlung. Marina trug den Entscheid in ihren eigenen Worten mit: «Ich gehe aber nie mehr ins Spital.» Marina spürte, was geschehen würde. "Mami, ich weiss nicht, wie ich in den Himmel komme», erklärte sie der Mutter. Regula Meier antwortete, dass die Kräuterhexe ihr den Weg weisen würde. Marina lächelte und sagte: «Dann bin ich morgen im Himmel.» In der folgenden Nacht zum ersten Mai 2013 verstarb Marina.

\section{Das Ende des Ausnahmezustandes}

Und plötzlich war der Ausnahmezustand weg. «Im ersten Jahr nach Marinas Tod kam es uns vor, als würden wir vom Leben weitergespült», schreibt Regula Meier im Buch. Das erste Jahr sei besonders schwierig, weil man mit den jüngeren Geschwistern alles zum ersten Mal, aber ohne die älteste Tochter mache - Ferien etwa oder Geburtstage feiern.

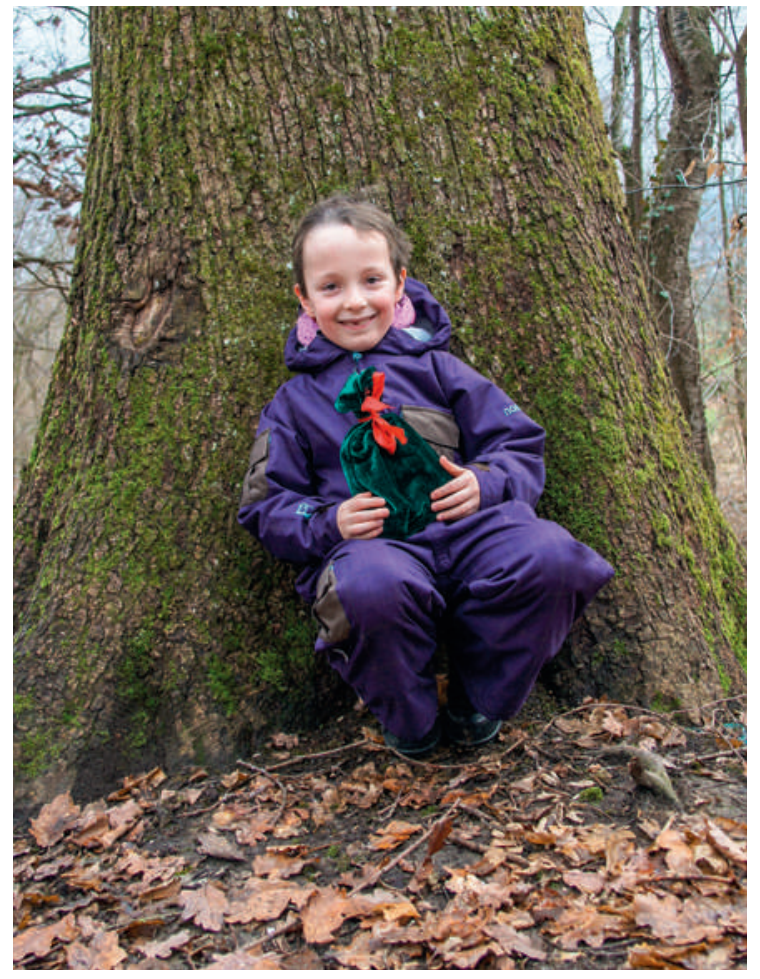

Marina hat gerade die Botschaft der Kräuterhexe gefunden ein Bild aus dem Buch von Marinas Mutter, das Eltern einen Weg zur Unterstützung ihrer kranken Kinder zeigen soll.

Auch nach Marinas Tod war die Kräuterhexe sehr wichtig, indem sie für die Geschwister die letzte Verbindung und Erinnerung an Marina war. Noch heute legt der inzwischen achtjährige Bruder Botschaften für die Kräuterhexe in den Wald - auch wenn er zu vermuten beginnt, Chrisanthemia könnte eine Erfindung der Mutter sein. Vor dem Schuleintritt von Marinas Bruder Till und dem Kindergarteneintritt ihrer Schwester Lilly schrieb die Kräuterhexe einen letzten Brief und erklärte darin auch, dass sie jetzt weiter müsse, um sich um andere schwerkranke Kinder zu kümmern.

\section{Die unmögliche Diagnose}

Familie Meier hat einen Weg gefunden, mit ihrer Trauer umzugehen, auch mithilfe der Phantasie: «Jeden Tag sprechen wir mit und von Marina, jeden Morgen zündet Till die Kerze neben ihrem Foto an und wir wünschen ihr einen tollen Tag», schreibt Regula Meier. Zur Unterstützung von ähnlich betroffenen Angehörigen gründete sie in Winterthur eine Gesprächsgruppe, die sich vier- bis sechsmal jährlich trifft. Das Angebot stösst auf Interesse bei Menschen, denen bisher die Möglichkeit fehlte, darüber zu sprechen. So besucht eine Mutter die Gruppe, deren Sohn bereits vor zehn Jahren verstorben ist. Bei anderen Eltern ist der Tod des Kindes erst wenige Wochen her. 


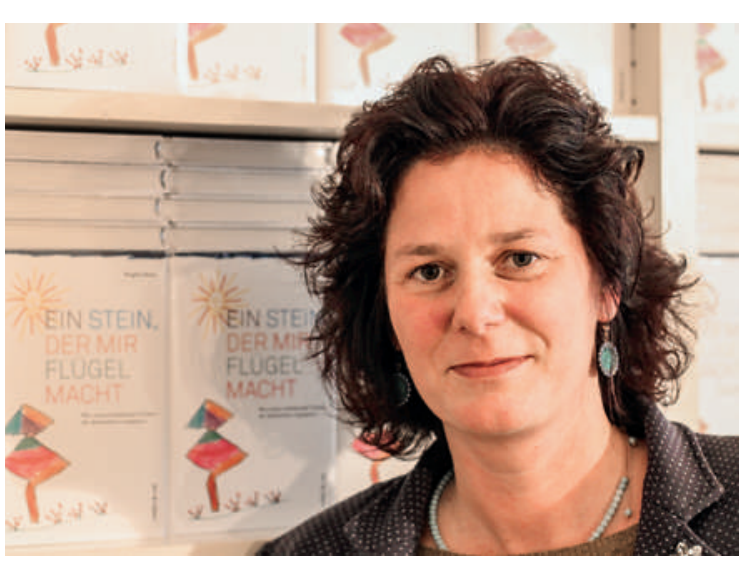

Viele Eltern erzählen Regula Meier von derselben Erfahrung: Sie wurden von den Ärzten bei den ersten Anzeichen nicht ernst genommen mit der Sorge, es könnte sich um eine gravierende Erkrankung halten. Oft würden sie mit der Diagnose «Verstopfung» oder "wachstumsbedingt» vertröstet, mit einem Abführmittel oder Schmerzmedikament nach Hause geschickt und die Krebserkrankung würde erst nach Umwegen diagnostiziert. So berichteten in der Gesprächsgruppe Eltern vom tragischen Fall ihres Kindes, bei dem von einem Zeckenstich die Rede war und nach dem plötzlichen Tod erst die Obduktion den Befund Lymphdrüsenkrebs ergab.

«Krebs bei einem Kind: Das scheint irgendwie nicht sein zu dürfen, passt nicht in unser Denken, dass Krebs vor allem ältere Menschen betrifft», sagt Regula Meier im Gespräch mit der Schweizerischen Ärztezeitung. Und doch erkranken rund 250 Kinder jährlich in der Schweiz an Krebs.

\section{Mit Mut zum Hund}

Wie hätte Marina und ihre Familie diese schwierige Zeit ohne Kräuterhexe überstehen können, fragte sich der Autor dieses Artikels bei der Lektüre des Buches von Regula Meier je länger je mehr. «Es ist auch für mich unvorstellbar, wie das hätte möglich sein sollen», sagt
Ein Buch, das zeigt, wie die Kräuterhexe half, wenn Marina fast verzweifelte und unter starken Schmerzen oder Nebenwirkungen der Therapie litt. Das Strahlen auf dem Gesicht der Tochter und die wiedererwachte Lebendigkeit schoben bei Regula Meier alle Zweifel beiseite, ob «dieser Schwindel überhaupt erlaubt sei».

Regula Meier

Ein Stein, der mir Flügel macht

Wie meine krebskranke Tochter der Kräuterhexe begegnete Zürich: rüffer \& rub Sachbuchverlag; 2015.

208 Seiten. $28.80 \mathrm{CHF}$.

ISBN 978-3-907625-89-7

die Autorin. Es ging irgendwie nicht mehr anders, als eine Phantasiefigur zu Hilfe zu holen. Andere Familien allerdings gehen ganz anders mit der Situation um, wie Regula Meier auf der Krebsstation im Spital erlebt hat mit Druck auf ihr Kind: Du musst diese Medikamente nehmen, du musst trinken. «Das wollten wir nicht. Und es entspricht nicht meiner Vorstellung einer kindgerechten Erziehung», so Regula Meier. Natürlich sei es nicht allen Eltern gegeben, solche Phantasiefiguren zu entwerfen. Auch seien nicht alle Kinder dafür empfänglich. Aber ihre Motivation, ein Buch zu schreiben, war es, Eltern einen möglichen Weg zu zeigen und Mut zu machen: Phantasie kann helfen, eine solch schwere Zeit zu überstehen und weiterzuleben.

Dass das Buch betroffenen Eltern Hoffnung macht, zeigen die Reaktionen darauf. An der Vernissage sagte ein junger Mann, der eine Krebserkrankung hinter sich hat, zu Regula Meier: «Ich hätte mir auch eine Kräuterhexe gewünscht.» Aber nicht nur für schwerkranke Kinder kann eine Phantasiefigur wie Chrisanthemia wertvoll sein. Eine Mutter schrieb Regula Meier in einem Brief von ihrem ängstlichen Kind. Der Junge getraute sich nicht, auf dem Weg zum Kindergarten an einem Hund vorbeizulaufen. Mit «Mutsteinen» funktioniere es jetzt.

Bildnachweis

Porträt R. Meier: Adrian Ritter; alle anderen: Regula Meier 